\title{
Visualizing in vitro-in vivo correlation of miktoarm copolymer nanomicelles in cancer cellular uptake and trafficking
}

\author{
Zheng Cuia,c, Xiaofei Zhanga,d, Xiaojin Zhang ${ }^{\mathrm{b}}$, Suna $\mathrm{He}^{\mathrm{a}, \mathrm{e}}$, Wei Gao,f, Bing $\mathrm{He}^{\mathrm{a}}$, \\ Xueqing Wang ${ }^{\mathrm{a}}$, Hua Zhang ${ }^{\mathrm{a}}$, Zhenlin Zhong ${ }^{\mathrm{b}}$, Qiang Zhang ${ }^{\mathrm{a}}$ \\ a Department of Pharmaceutics, School of Pharmaceutical Sciences, Peking University, \\ Beijing 100191, China \\ ${ }^{b}$ Key Laboratory of Biomedical Polymers of Ministry of Education, College of Chemistry \\ and Molecular Sciences, Wuhan University, Wuhan 430072, China
}

\begin{abstract}
In this study, a clear correlation between the in vitro and in vivo cellular uptake and trafficking was discovered by delivering miktoarm copolymer nanomicelles (MCNs) to cancer cells and tumor tissues. To monitor this process, two different FRET pairs, DiO and Dil, DiD and DiR, were loaded into MCNs to monitor the Förster resonance energy transfer (FRET) efficiency. The change in FRET efficiency in vitro and in vivo demonstrated a similar sequence of events for the transport of MCNs: hyperbranched block PCL inserted into cytomembrane, while the loaded hydrophobic fluorescence probes were released and followed by time-dependent intracellular clustering within endocytic vesicles. Additionally, uptake of loaded fluorescence probes with successively increasing ratios of copolymers suggested that with the increase of mass ratio of copolymer to fluorescence probes, cellular uptake of probes significantly decreased. This result was also consistent with the uptake behavior in cancer tissues. Collectively, the interaction between MCNs and cellular membrane dictated the uptake and trafficking of core-loaded hydrophobic probes. This concept paves a new way to analyze in vitro-in vivo correlation of other nanocarriers for endocytosis mechanism studies as well as further novel copolymers design in biomedical applications.
\end{abstract}

\section{Keywords:}

miktoarm copolymers ' nanomicelles cellular uptake - intracellular trafficking FRET· in vitro-in vivo correlation

Advances in nanotechnology have fostered to construct a diversity of nanomaterials with the ability to encapsulate and transport therapeutic or diagnostic agents ${ }^{1-3}$. Among these nanoscale delivery vehicles, polymeric micelles attracted the most attention in diverse biomedical fields as drug delivery vehicles ${ }^{4-5}$, contrast agents carriers ${ }^{6-7}$ and diagnostic devices ${ }^{8-9}$. The advantages of polymeric micelles used as drug delivery vectors include improving the solubility of poorly water soluble drugs with the amphiphilic structures ${ }^{10}$, stabilizing and protecting drugs that are sensitive to the surrounding environment ${ }^{11}$, reducing nonspecific uptake by the reticuloendothelial system (RES) $)^{12}$ and achieving the passive targeting delivery in solid tumors ${ }^{13-14}$. The conventional micelle drug delivery systems are mainly based on linear amphiphilic copolymers. However, linear amphiphilic copolymer micelles will dissemble in vivo when the concentration of the copolymer is diluted by the bloodstream to fall below the critical 
micelle concentration (CMC). As a result, the instability of linear amphiphilic copolymer micelles greatly limits their clinical application ${ }^{15}$.

To overcome the disadvantage of classical micelles, recent advances in synthetic methodologies have fostered to develop amphiphilic polymers with more complex architectures, including dendrimers, hyperbranched polymers, cyclic polymers, and star polymers $^{15-16}$ in order to increase capacity of copolymer micelles and to enhance the stability of micelles in vivo. In this paper, we used novel amphiphilic miktoarm copolymers $\mathrm{PEG}_{113}-(h b-\mathrm{PG})_{15}-\mathrm{g}-\mathrm{PCL}_{22}$ with a linear-hyperbranched architecture bearing one monomethoxy poly(ethylene glycol) (mPEG) chain and several poly( $\varepsilon$-caprolactone) (PCL) chains on a hyperbranched polyglycerol ( $h b-P G)$ core $^{17}$ (Figure 1A) to construct ultrastable miktoarm copolymer nanomicelles (MCNs). The highly hydrophobic branched structure of PCL chains offers inner porosity and imposes a robust steric barrier for embedded water insoluble drugs, which at the same time potentially allowing good release control. PEGylation is a common strategy to increase water solubility and compatibility in polymeric delivery systems. Moreover, densely PEG-coated MCNs enable their long circulation in vivo by evading the reticuloendothelial system (RES) ${ }^{18}$.

Hitherto, many drug-incorporated polymeric micelles are currently undergoing clinical trials ${ }^{19-21}$, which contributes to our understanding of the pathways involved in polymeric micelle delivery ${ }^{22}$. However, to our knowledge, no studies have examined the cellular uptake mechanism of miktoarm copolymer micelles. Furthermore, despite a great deal of insight into the endocytosis mechanism of classical micelles in vitro ${ }^{23-24}$, in vitro-in vivo correlation of nanomicelle transport is still unknown because no nanoscale direct visualization of how the nanomicelles are internalized and transported have been shown. Most current studies of intracellular trafficking of nanomicelles are mainly limited to colocalization of nanomaterials with specific endocytic markers or the exclusion of specific mechanisms by chemical inhibition or cell mutation ${ }^{25-26}$.

In an attempt to address these problems mentioned above, we investigated cancer cellular uptake and trafficking correlation of $\mathrm{PEG}_{113}-(h b-\mathrm{PG}){ }_{15}-g-\mathrm{PCL}_{22} \mathrm{MCNs}$ in vitro and in vivo. The basic concept of the entire study was illustrated in Figure $1 \mathrm{~B}$. The current work characterized the physical properties of MCNs, tested their internalization modalities of dual-labeled MCNs, visualized the release and transport process with in vitro and in vivo FRET imaging, and began to determine their underlying internalization mechanisms employing successively increasing ratio of copolymers. We show here that cellular uptake of loaded hydrophobic probes is much faster than labeled copolymers. The transport process in vitro and in vivo involved a clear sequence of events: hyperbranched block PCL inserted into the cytomembrane, while the loaded hydrophobic fluorescence probes released, followed by time-dependent intracellular clustering within endocytic vesicles. With the increase of mass ratio of copolymer, the uptake amount and speed of loaded fluorescent probes significantly decrease, which resulted in slower in vivo clearance rates of loaded fluorescent probes in the peripheral region of the tumor.

The reason for choosing these four kinds of fluorescent probes (DiO, Dil, DiD and $\mathrm{DiR}$ ) in this study was as follows. (1) DiO, Dil, DiD and DiR are all hydrophobic fluorescence probes and thus can be used to represent hydrophobic drugs loaded in MCNs. (2) The fluorescent probes, DiO and Dil, are used in cellular assays, while the near-infrared fluorescent probes DiD and DiR-loaded MCNs are used in animal studies. 
In this case, the similar chemical structures of DiO, Dil, DiD and DiR could aid us to construct dye-loaded MCNs with similar release behavior in order to discover the in vitro -in vivo correlation. (3) The FRET pair DiO and Dil, with DiO as donor and Dil as acceptor, was used to monitor FRET efficiency in vitro; while the second FRET pair DiD and DiR, with DiD as donor and DiR as acceptor was used to detect the change of FRET efficiency in vivo.

\section{Materials and methods}

Materials. $\mathrm{PEG}_{113}-(h b-\mathrm{PG})_{15-}-\mathrm{P}-\mathrm{PCL} 22(\mathrm{Mn}=8500)$ was kindly donated by $\mathrm{Dr}$. Zhenlin Zhong (Wuhan University) ${ }^{17}$. Sulforhodamine B (SRB), trichloroacetic acid (TCA) and Tris base were all purchased from Sigma-Aldrich (St. Louis, MO, USA). Hoechst 33258 was purchased from Molecular Probes, Inc (Oregon, USA). DiO and Dil were provided by Beyotime Institute of Biotechnology (Shanghai, China); DiD and DiR were purchased from Biotium, Inc (Hayward, USA).

Preparation of DiO or/and Dil, DiD or/and DiR-loaded MCNs. DiO and Dil, DiD and $\mathrm{DiR}$ were chosen as FRET pairs for the following study. All the micelles were prepared using thin film hydration method ${ }^{27}$. Briefly, dyes (DiO, Dil, DiD, DiR), PEG-(hb-PG)-gPCL were codissolved in acetonitrile. The acetonitrile was evaporated under vacuum with a rotary evaporator at $40{ }^{\circ} \mathrm{C}$. The obtained copolymer film was hydrated in RPMI1640 medium, followed by stirring in the $40{ }^{\circ} \mathrm{C}$ water bath for $5 \mathrm{~min}$. Finally, the solution was filtered through a $0.22 \mu \mathrm{m}$ membrane. The mass ratio of dye to copolymer was 1:500, 1:750, 1:1500 and 1:3000. The final concentration of DiO or Dil for the cell uptake assay was $2 \mu \mathrm{g} / \mathrm{mL}$.

Characterization of DiO- or/and Dil-, DiD- or/and DiR-loaded MCNs. The particle size and zeta potential of the prepared blank MCNs and dye-loaded MCNs were measured by dynamic light scattering (DLS) using Malvern Zetasizer Nano ZS (Malvern, UK) at $25{ }^{\circ} \mathrm{C}^{28}$. The morphology of blank MCNs after dilution was investigated by transmission electron microscope (TEM, JEOL, Japan) after negative staining with uranyl acetate solution (1\%, w/v). Encapsulation efficieny (E.E) was defined as the actual amount of fluorescent probes encapsulated as detected by the fluorospectrophotometer divided by the original amount of fluorescent probes. The probe-loaded micelles were diluted by acetonitrile and the concentration of fluorescence probes was measured using fluorospectrophotometer ${ }^{29}$.

The critical micelle concentration (CMC) was determined using pyrene as a fluorescent probe ${ }^{4}$. The concentration of block copolymer varied from $0.05 \mu \mathrm{g} / \mathrm{mL}$ to 50 $\mu \mathrm{g} / \mathrm{mL}$ and the concentration of pyrene was fixed at $6 \times 10^{-6} \mathrm{M}$. The fluorescence spectra were recorded using a Cary Eclipse fluorescence spectrometer using an emission wavelength of $390 \mathrm{~nm}$. The excitation spectra were recorded ranging from $300 \mathrm{~nm}$ to $360 \mathrm{~nm}$. The emission fluorescence at $333 \mathrm{~nm}$ and $335 \mathrm{~nm}$ was monitored. The CMC was estimated as the cross point when extrapolating the intensity ratio $I_{335} / I_{333}$ at low and high concentration regions.

Cell Culture. Human breast cancer MCF-7 cells were obtained from the Institute of Basic Medical Science, Chinese Academy of Medical Sciences (Beijing, China). Cells were cultured in RPMI-1640 medium (M\&C Gene Technology, Beijing, China) supplemented with $10 \%$ fetal bovine serum (FBS), 100 units/mL penicillin and 100 $\mu \mathrm{g} / \mathrm{mL}$ streptomycin at $37{ }^{\circ} \mathrm{C}$ in a humidified atmosphere containing $5 \% \mathrm{CO}_{2}$. The cells 
for all experiments were in the logarithmic phase of growth ${ }^{30}$.

Cytotoxicity assay. The cytotoxicity of MCNs was evaluated in vitro using the SRB (sulforhodamine B) and LDH (lactate dehydrogenase) assays. SRB assay was performed on MCF-7 cells as originally described. Briefly, MCF-7 cells were seeded into 96-well culture plates at $3000-3500$ cells/well and grown at $37{ }^{\circ} \mathrm{C}$ in the presence of $5 \%$ $\mathrm{CO}_{2}$ for $24 \mathrm{~h}$. The final concentration of MCNs was in the range of $0.025-6 \mathrm{mg} / \mathrm{mL}$. The culture medium without any MCNs was used as a blank control. 9\% Triton X-100 was added as a positive control. After exposure for $24 \mathrm{~h}$, the cells were fixed with $10 \%$ trichloracetic acid (TCA) at $4{ }^{\circ} \mathrm{C}$ for $1 \mathrm{~h}$, the 96 -well plate was then washed 4 times with water, while vigorously flicking the plate between washes to remove excess water. SRB solution was added into each well and incubated for $30 \mathrm{mins}$, after which the excess dye was removed by washing repeatedly with $1 \%$ acetic acid. After air-drying, the bound dye was re-dissolved in $10 \mathrm{mM}$ Tris base solution for OD determination at $565 \mathrm{~nm}$ using a microplate reader (Bio-Rad 680, America).

As an additional test, we also measured the leakage of LDH in the culture medium. The assay relies on measuring the activity of $\mathrm{LDH}$ in catalyzing the reaction: $N A D^{+}+$Lactate $\stackrel{L D H}{\longleftrightarrow} N A D H+$ Pyruvate. The culture medium without any MCNs was used as a blank control. $9 \%$ Triton X-100 was added as a positive control. After treatment with MCNs (6 mg/mL, $1 \mathrm{mg} / \mathrm{mL}$ ) for $24 \mathrm{~h}$, media were collected and centrifuged, and the activity of LDH released from the cytosol of damaged cells was assessed using the LDH Cytotoxicity Assay Kit (Applygen Technologies Inc, China). The maximum releasable LDH activity in the cells, induced by the addition of $9 \%$ Triton $X$ 100 , was measured and used as $100 \%$ LDH released. The optical density at a wavelength of $440 \mathrm{~nm}$ was measured using a microplate reader (Bio-Rad 680, America). The formula below was used to calculate LDH release percentage.

$$
\% \text { LDH Release }=\frac{\left|O D_{\text {sample }}-O D_{\text {blankcontrol }}\right|}{\left|O D_{\text {TritonX-100 }}-O D_{\text {blankcontrol }}\right|} \times 100
$$

Flow cytometry analysis. Approximately $6.0 \times 10^{5} \mathrm{MCF}-7$ cells/well were seeded in 6-well plates and cultured for $24 \mathrm{~h}$ at $37^{\circ} \mathrm{C}$. Cells were exposed to various $\mathrm{DiO}$ and Dil co-loaded MCNs (the mass ratio of dyes and copolymers were 0, 1:500, 1:750, 1:1500 and 1:3000, respectively) at $37^{\circ} \mathrm{C}$ for $1 \mathrm{~h}$ (or longer periods as indicated in the legend) for cellular internalization studies. Concentration of DiO and Dil in all formulations was 2 $\mu \mathrm{g} / \mathrm{mL}$. Cells were then washed and trypsinized, centrifuged, resuspended in pre-cooled PBS. Flow cytometry was performed on a FACScan flow cytometer (FACScan, Becton Dickinson, San Jose, CA).

Spectroscopic characterization of dyes. The emission spectra were recorded on a Cary Eclipse ultraviolet/visible light/near-infrared spectrophotometer (Varian Inc.) using 1-cm quartz cells. Samples included DiO-loaded MCNs (DiO MCNs), Dil-loaded MCNs (Dil MCNs), DiO- and Dil-co-loaded MCNs (DiO/Dil MCNs), DiO-loaded MCNs mixed with Dil-loaded MCNs (DiO MCNs \& Dil MCNs Mixtures), and DiO/Dil MCNs destroyed by $10 \times$ acetonitrile. The samples above were excited at $484 \mathrm{~nm}$, and the emission spectra of the donor/acceptor were recorded at $490-600 \mathrm{~nm}$ for FRET measurements. Moreover, samples including DiD-loaded MCNs (DiD MCNs), DiR-loaded MCNs (DiR MCNs), DiD- and DiR-coloaded MCNs (DiD/DiR MCNs), and DiD-loaded MCNs mixed with DiR-loaded MCNs (DiD MCNs \& DiR MCNs Mixtures) were excited at $644 \mathrm{~nm}$ and 
the emission spectra of the donor/acceptor recorded at $650-800 \mathrm{~nm}$ for FRET measurements.

Intracellular monitoring fate of hydrophobic probes using FRET. To monitor the fate of hydrophobic dyes released from MCNs, MCF-7 cells were treated with DiO MCNs, Dil MCNs, DiO/ Dil MCNs and DiO MCNs \& Dil MCNs Mixtures in the serumfree 1640 medium, respectively. After incubation at predetermined time intervals, cells were washed twice with pre-cooled PBS before they were fixed in $4 \%$ paraformaldehyde. The fixed cells were visualized and examined using a Leica TCS SP5 confocal microscope. Confocal images were acquired with the excitation at $488 \mathrm{~nm}$. The emission wavelength was between 555-655 nm for Dil (Acceptor) detection and the emission wavelength was between $500-530 \mathrm{~nm}$ for DiO (Donor) detection.

Tumor Implantation. Female BALB/c nude mice $(18-20 \mathrm{~g})$ were purchased from Vital Laboratory Animal Center (Beijing, China) and acclimated at $25^{\circ} \mathrm{C}$ and $55 \%$ humidity under natural light/dark conditions for 1 week before the study, with free access to standard food and water (Vital Laboratory Animal Center, Beijing, China). MCF-7 tumorbearing mice were prepared by inoculating $4 \times 10^{6} \mathrm{MCF}-7$ cells in the right flank of female $\mathrm{BALB} / \mathrm{c}$ nude mice. When tumor volume reached about $300 \mathrm{~mm}^{3}$, MCF-7 tumor bearing mice were randomly assigned to groups for live imaging studies. All procedures were approved by the Institutional Animal Care and Use Committee at Peking University Health Science Center and were in accordance with international guidelines on the ethical use of animals ${ }^{31-32}$.

In vivo monitoring the uptake and trafficking of hydrophobic NIRF FRET probes. To verify the role of MCNs in delivering hydrophobic molecules to xenografted MCF-7 tumors, hydrophobic NIRF FRET probes (DiD, DiR) were utilized. Animal model of female BALB/C mice xenografted MCF-7 tumors were established in the same way as discussed above. Animals in two groups received the following two treatments respectively: DiD/DiR MCNs and DiD MCNs \& DiR MCNs Mixtures. The dose of DiD and DiR in this study was $100 \mu \mathrm{g} / \mathrm{kg}$. Near-infrared fluorescence(NIRF) imaging experiments were performed using a Kodak multimodel imaging system (Carestream Health, Inc. USA) at $1 \mathrm{~h}, 3 \mathrm{~h}, 5 \mathrm{~h}, 8 \mathrm{~h}, 12 \mathrm{~h}$ and $24 \mathrm{~h}$. FRET images were acquired with an excitation bandpass filter at $610 \mathrm{~nm}$ and an emission bandpass filter at $790 \mathrm{~nm}$. DiD images were acquired with an excitation bandpass filter at $610 \mathrm{~nm}$ and an emission bandpass filter at $720 \mathrm{~nm}$. DiR images were acquired with an excitation bandpass filter at $720 \mathrm{~nm}$ and an emission bandpass filter at $790 \mathrm{~nm}$. Fluorescence exposure time was 2 min and X-Ray exposure time was $30 \mathrm{~s}$ per image. All images were normalized and analyzed using the Carestream MI SE software.

In vivo distribution studies by live imaging studies. In order to observe the realtime distribution and tumor accumulation ability of various fluorescence DiR MCNs formulations in PBS, including the mass ratio of DiR to copolymers of 1:500, 1:750, 1:1500 and 1:3000, non-invasive in vivo imaging systems were utilized on the female $\mathrm{BALB} / \mathrm{c}$ mice xenografted MCF-7 tumors. The mice were injected with $0.2 \mathrm{~mL}$ of various $\mathrm{DiR}$ MCNs listed above $(100 \mu \mathrm{g} / \mathrm{kg}$ DiR) respectively, via the tail vein, and then anesthetized by $2 \%$ isoflurane delivered via a nose cone system before scanning. Nearinfrared fluorescence (NIRF) imaging experiments using a Kodak multimodel imaging system (Carestream Health, Inc. USA) at 5 h, 8 h, 12 h, 24 h, 48 h, 60 h, 72 h and 96 h with an excitation bandpass filter at $720 \mathrm{~nm}$ and an emission bandpass filter at $790 \mathrm{~nm}$. 
Fluorescence exposure time was $2 \mathrm{~min}$ and X-Ray exposure time was $30 \mathrm{~s}$ per image.

Intratumoral distribution of hydrophobic probes. To observe dye uptake and distribution into MCF-7 tumors, female BALB/c mice xenografted MCF-7 tumors were administered DiO/ Dil MCNs, DiO MCNs and Dil MCNs Mixtures in PBS via injection into the tail vein $(100 \mu \mathrm{g} / \mathrm{kg} \mathrm{DiR})$. The mass ratio of $\mathrm{DiO}$ and Dil to copolymers was 1:750. The concentration of DiD and DiR was $100 \mu \mathrm{g} / \mathrm{kg}$. After $12 \mathrm{~h}$, the mice were sacrificed, and tumor tissues were harvested, placed in Tissue-Tek OCT embedding medium (Sakura Finetek, Tokyo, Japan), frozen on dry ice, sectioned using a cryostat, mounted on slides, and then fixed in $4 \%$ paraformaldehyde. The sections were incubated with Hoechst $33258(1 \mu \mathrm{g} / \mathrm{mL})^{33}$ for $30 \mathrm{~min}$ at room temperature followed by washing with PBS. The stained tumor cryosections were examined using CLSM (Leica TCS SP5).

\section{Results and discussion}

Characterization of MCNs. Miktoarm copolymers have received increased attention because of their stability in forming nanomicelles and flexibility in their synthesis for biomedical applications. In this study, we used amphiphilic miktoarm copolymers $\mathrm{PEG}_{113-}(h b-\mathrm{PG})_{15}-\mathrm{g}$-PCL22 to construct nanomicelles. Figure 1 shows the schematic illustration of self-assembled MCNs. The hydrophobic PCL segments are locked in the dense inner core of micelles, while the hydrophilic PEG and PG chains formed the corona shell. MCNs encapsulated with five different combinations of hydrophobic fluorescence probes were prepared and characterized. The hydrodynamic diameters of the five MCNs with different encapsulations were approximately $50 \mathrm{~nm}$ with polydispersity indices (PDI) well below 0.22 (Table S1, Figure 1). The zeta potential of five MCNs was all around $0 \mathrm{mV}$, which indicated that encapsulation did not affect the zeta potential of MCNs. The morphology of spherical DiO and Dil co-loaded with MCNs (DiO/Dil MCNs) was observed in the TEM image (Figure 1). All encapsulation efficiencies of the five MCNs were around $90 \%$ (Table S1), meaning that MCNs have great potential in loading water-insoluble drugs if used to construct drug delivery systems. According to the excitation spectra of pyrene at different polymer concentrations, the CMC of $\mathrm{PEG}_{113}-(h b-\mathrm{PG})_{15}-g-\mathrm{PCL}_{22}$ miktoarm copolymer nanomicelles was determined to be $0.37 \mu \mathrm{g} / \mathrm{mL}$.

Cytotoxicity test for MCNs. Cytotoxicity is a main concern in the application of drug delivery systems. The commonly used MCF-7 breast cancer cells were chosen for this study and the following in vivo assays. To examine the cytotoxicity of MCNs, we incubated MCF-7 cells with MCNs for $24 \mathrm{~h}$. The toxicity of MCNs to MCF-7 cells was measured by the SRB assay ${ }^{34}$. Beyond our expectations, MCNs increased cell viability slightly in all groups as shown in Figure S1A. No statistically significant differences in toxicity were observed between the groups treated with different concentrations of MCNs samples. The LDH assay is a method of measuring the membrane integrity as a function of the amount of cytoplasmic LDH leaked into the medium ${ }^{35}$. To test membrane rupture, LDH assay was utilized on MCF-7 cells. $1 \mathrm{mg} / \mathrm{mL}$ and of $6 \mathrm{mg} / \mathrm{mL} \mathrm{MCNs}$ used in our following cellular assay was tested in the LDH assay. The LDH leakage in the medium for the two groups did not significantly increase $(P>0.05$, Figure $S 1 B)$. These results indicated that there was no significant cytotoxicity observed for the MCNs on MCF-7 cells. 
Intracellular trafficking studies of FRET MCNs on MCF-7 cells. A) FRET measurements. To monitor the release process of core-loaded hydrophobic molecules from MCNs in vitro, FRET pair DiO (Donor) and Dil (Acceptor) co-loaded MCNs (DiO/Dil MCNs) were prepared. The FRET of $\mathrm{DiO} / \mathrm{Dil} \mathrm{MCNs}$ was investigated with a fluorospectrophotometer. Figure 2A shows the fluorospectra of MCNs loaded with $\mathrm{DiO}$ (DiO MCNs), MCNs loaded with Dil (Dil MCNs), DiO/Dil MCNs, mixtures of MCNs loaded with $\mathrm{DiO}$ and MCNs loaded with Dil (DiO MCNs \& Dil MCNs Mixtures). Energy transfer was evaluated by the intensity ratio of acceptor to donor $\left(/_{\mathrm{Dii}} / \mathrm{L}_{\mathrm{DiO}}\right)$, calculating the intensities of the acceptor emission $(565 \mathrm{~nm})$ and the intensities of the donor emission $(501 \mathrm{~nm})$ using the excitation at $484 \mathrm{~nm}$. For DiO/Dil MCNs, DiO and Dil was condensed together by MCNs, with a FRET ratio of 2.40. When DiO-loaded MCNs and Dil-loaded MCNs were prepared respectively and mixed with each other (DiO MCNs \& Dil MCNs Mixtures), no FRET existed with a FRET ratio of 0.25 . DiO and Dil were not directly condensed with each other, thus FRET could not occur. After DiO/Dil-loaded MCNs decomposed by acetonitrile, the FRET signal disappeared because $\mathrm{DiO}$ and Dil could not be closely condensed any more, resulting in a FRET ratio of 0.08 . Figure $2 \mathrm{~B}$ shows the CLSM image of DiO/Dil loaded MCNs and its decomposition by acetonitrile. For DiO/Dil MCNs, the fluorescence intensity of the donor DiO strongly decreased in the presence of the acceptor Dil, and simultaneously, the emission of Dil was enhanced. When decomposed by acetonitrile, the fluorescence intensity of the donor DiO recovered. At the same time, FRET-mediated Dil emission strongly decreased.

B) FRET efficiency comparison between FRET Sensitized Emission (SE) method and FRET Acceptor Photobleaching (AB) method. A proper method to analyze the FRET efficiency in MCF-7 cells was required to evaluate the endocytosis behavior of MCNs. Sensitized Emission (SE) is one of the most popular methods for evaluation of FRET efficiencies. This method involves measuring the donor and the FRET signal (donor excitation only) in sequence with the detection of the acceptor (acceptor excitation only ${ }^{36}$. FRET Acceptor Bleaching is another frequently used method for FRET efficiency measurements in vitro. The method involves measuring the donor "dequenching" in the presence of an acceptor. This can be done by comparing donor fluorescence intensity in the same sample before and after destroying the acceptor by photobleaching. If FRET was initially present, a resultant increase in donor fluorescence will occur after photobleaching the acceptor ${ }^{37}$. Both methods were used to evaluate FRET efficiency in MCF-7 cells $4 \mathrm{~h}$ post-DiO-Dil MCNs incubation. Five circled regions were selected accordingly to measure the FRET efficiency by both the FRET SE method (Figure S2A) and FRET AB method (Figure S2B). After t- test calculation with a significance level under $5 \%$, there was not enough evidence to show that data obtained from the two methods were different. Considering that the FRET AB method required 8 $s$ to photobleach each region before measuring FRET efficiencies, the FRET SE method is less time-consuming if dealing with the same amount FRET samples. Moreover, it is possible to bleach other regions accidentally if frequently photobleaching each spot and introduce error in the FRET efficiency measurement. As a result, the FRET SE method was utilized to monitor the dynamics of cellular endocytosis in the following experiment.

C) Intracellular fate of FRET MCNs in MCF-7 cells. We continued to investigate the intracellular fate of two kinds of MCNs (DiO/Dil MCNs and DiO MCN \& Dil MCN 
Mixtures) in MCF-7 cells using FRET imaging. CLSM images showed the representative intracellular fate of DiO/Dil MCNs and DiO MCNs \& Dil MCNs Mixtures in the majority of the cells (Figure 3). The FRET SE method was used to quantify FRET efficiencies by the spectra intensities recorded from 30 fluorescent areas inside the cells. By incubating MCF-7 cells with $\mathrm{DiO} / \mathrm{Dil} \mathrm{MCNs}$ at $37^{\circ} \mathrm{C}$ for $1 \mathrm{~h}$, we observed the significantly diminished FRET effect (Figure 3A). The presence of co-localization of DiO and Dil but absence of FRET fluorescence from the plasma membrane might suggest a release of the two fluorescent probes from MCNs during cellular endocytosis of MCNs. It is also possible that not enough micelles were uptaken by cells within the short incubation time. At $2 \mathrm{~h}$ and $4 \mathrm{~h}$ incubation, the fluorescence signals of FRET-mediated Dil became stronger. The results indicated that $\mathrm{DiO}$ and Dil were likely released from MCNs and concentrated in endocytic vesicles in the process of cellular endocytosis and intracellular sorting, which led to the recovery of FRET signals. More interesting results were observed from the group of DiO MCNs \& Dils MCNs Mixtures (Figure 3B). DiO MCNs \& Dil MCNs Mixtures had no FRET signals because of separate encapsulation of the two fluorescence probes. However, after incubating MCF-7 cells with DiO MCNs \& Dil MCNs Mixtures for $2 \mathrm{~h}$ and $4 \mathrm{~h}$ at $37{ }^{\circ} \mathrm{C}$, the FRET efficiencies were almost the same with that of the DiO/Dil MCNs' (Figure 3C). The results from both groups further proved that $\mathrm{DiO} / \mathrm{Dil} \mathrm{MCNs}$ and $\mathrm{DiO}$ MCNs \& Dil MCNs Mixtures have similar pathways in cellular trafficking.

Taken together with the cellular uptake study in Figure 3, the current results possibly indicated that the internalization speed of miktoarm copolymers was slower than that of core-loaded hydrophobic probes. This probably resulted from the hyperbranched hydrophobic block PCL, which easily embedded into cell membranes but was hard to be transferred into cells. Another proposition was that the PEG shell of $P_{E G}{ }_{113}-(h b-P G)_{15-}-$ $\mathrm{PCL}_{22}$ miktoarm copolymer would facilitate the fast release of its core loaded hydrophobic probes as result of PEG bridging between the phospholipid membranes of its adjacent cells ${ }^{38}$. While MCNs released the core-loaded hydrophobic probes into cells, the probes were re-encapsulated into endocytic vesicles and transported into the cells.

In vivo trafficking studies of FRET MCNs on MCF-7 tumor-bearing mice. A) FRET measurements. To monitor the dynamics of MCNs transferring in vivo, two nearinfrared carbocyanine dyes DiD and DiR were loaded into MCNs. The chosen dyes DiD (donor: excitation [ex] /emission [em], 644/665 nm) and DiR (acceptor: excitation [ex] lemission [em], 750/780 nm) display a spectral overlap between the donor emission and the acceptor excitation spectrum ${ }^{39}$. Moreover, the near infrared excitation and emission wavelength of both $\mathrm{DiD}$ and $\mathrm{DiR}$ could effectively reduce the interference of animal auto-fluorescence in the following in vivo study ${ }^{40}$. Figure 4A shows the fluorospectra of MCNs loaded with DiD (DiD MCNs), MCNs loaded with DiR (DiR MCNs), MCNs loaded with DiD and DiR (DiD/DiR MCNs) and mixtures of DiD MCNs and DiR MCNs (DiD MCNs \& DiR MCNs Mixtures). To certify the occurrence of FRET, all samples were excited at $644 \mathrm{~nm}$. For DiD/DiR MCNs, a strong DiR signal was observed due to the close proximity of DiD and DiR in the core, with a FRET ratio of $1.25\left(\mathrm{I}_{\mathrm{DiR}} / \mathrm{I}_{\mathrm{DiD}}\right)$. No FRET signal was observed in the sample of DiD MCNs \& DiR MCNs Mixtures, with a FRET ratio of 0.12 . Figure $4 \mathrm{~B}$ shows in vivo NIR fluorescence imaging of these respective preparations. Upon excitation at $644 \mathrm{~nm}$, strong emission of DiR FRET-mediated by DiD indicated compact encapsulation of DiD and DiR in same MCNs. In comparison, DiD 
MCNs \& DiR MCNs Mixtures have only a slight emission in the FRET channel because DiD and DiR was not directly condensed with each other, but associated with MCNs respectively.

B) In vivo fate of FRET MCNs on MCF-7 tumor-bearing mice. In vivo fate of DiD/DiR MCNs and DiD MCNs \& DiR MCNs Mixtures was studied on MCF-7 xenografts in nude mice by NIR fluorescence imaging. As shown in Figure 5A, from $1 \mathrm{~h}$ to $8 \mathrm{~h}$, the FRET- mediated DiR fluorescence intensity in DiD/DiR MCNs group was significantly higher than that of the DiD MCNs \& DiR MCNs Mixtures group. Consistently, the DiD (donor) fluorescence intensity in DiD/DiR MCNs group was significantly lower than that of the DiD MCNs \& DiR MCNs Mixtures group. This indicates the integrity of the DiD/DiR MCNs and the donor DiD transfers the excitation energy to the acceptor DiR due to the close proximity. From $8 \mathrm{~h}$ to $24 \mathrm{~h}$, the fluorescence intensity of DiD and that of FRET-mediated DiR was almost the same in both DiD/DiR MCNs and DiD MCNs \& DiR MCNs Mixtures groups. The FRET-mediated DiR fluorescence intensity achieved its highest level at $24 \mathrm{~h}$. This observation demonstrated that in the first or tissue phase (1-8 h), our constructed MCNs accumulated, but were not internalized by tumor tissue through the EPR effect. As a result, fluorescence intensity of FRET-mediated DiR in the DiD/DiR MCNs group was higher than that of the DiD MCNs \& DiR MCNs Mixtures group. In the second or cellular phase (8-24 h), we can infer the following process from the observation. 1) Similar to the first phase, more MCNs slowly accumulated in tumor tissue and ultimately reached the highest level at 24 h. 2) More importantly, MCNs were gradually internalized into MCF-7 tumor cells in this phase. Consistent with the result from trafficking studies in vitro (Figure 3A), fluorescent probes in MCNs were most likely concentrated in endocytic vesicles in the process of cellular internalization and intracellular sorting. FRET will take place in the concentrated DiD/DiR endocytic vesicles, same as FRET observed in DiD/DiR MCNs. Consequently, in the case of DiD MCNs \& DiR MCNs Mixtures, the FRET-mediated DiR intensity gradually reached the same level with that of the DiD/DiR MCNs group in the second phase.

In tumor tissues, the distribution of $\mathrm{DiO}$ and Dil in tumor cells were subsequently examined with CLSM after tail vein injection of either DiO/Dil MCNs or DiO MCNs \& Dil MCNs Mixtures. The distribution accorded with the FRET imaging and analysis by NIR fluorescence imaging (Figure 5B). Collectively, the in vitro and in vivo trafficking studies confirmed the correlation of cellular endocytosis process of MCNs loaded with hydrophobic fluorescent probes.

Cellular uptake of MCNs with different copolymer mass ratios. After the clarification of the MCN trafficking pathway in vitro and in vivo, the underlying interaction mechanism of MCNs with the cell membrane was assessed by comparing the uptake amount and speed of different MCNs. Four kinds of DiO/Dil MCNs were prepared and the mass ratio of copolymer to DiO/Dil probes was 500:1, 750:1, 1500:1 and 3000:1. Our results suggested that with the increase of mass ratio of copolymer, the uptake of $\mathrm{DiO}$ and Dil significantly decreased (Figure 6). Similar observations were noticed in both flow cytometry (Figure 6A, 6B) analysis and CLSM studies (Figure 6C). This result suggested that the mass ratio of the copolymer affected internalization of the hydrophobic fluorescence probes.

To further investigate factors affecting cellular uptake, we calculated the uptake speed of three kinds of DiO/Dil MCNs (the uptake of 3000:1 mass ratio of copolymer to 
$\mathrm{DiO} / \mathrm{Dil}$ probes MCNs was too insignificant to be calculated and thus wasn't included in further studies). The fluorescence intensity of DiO or Dil in each group was plotted with time points. As indicated in Figure 6B, three groups all showed burst-free uptake in a linearly increasing. This suggested that different MCNs interacted with cells possibly via the same endocytosis pathway.

Moreover, in order to further assess the influence of copolymers, we pre-incubated MCF-7 cells with blank MCNs (MCNs without loading fluorescent probes) for $1 \mathrm{~h}$, copiously washed the cells with PBS, and incubated them with DiO/Dil MCNs (the mass ratio of copolymer to DiO/Dil probes is $500: 1$ ) for another $2 \mathrm{~h}$. It was found that the uptake of DiO and Dil decreased after pre-incubation with blank MCNs (Figure 6D), presumably suggesting that the amphiphilic hyperbranched copolymers might embed into the cell membrane after preincubation, which affected the fluidity of the cell membrane and therefore hindered the subsequent cellular uptake of DiO/Dil MCNs.

In vivo distribution of DiR MCNs with different copolymer ratios. To develop a correlation between in vitro cellular uptake and in vivo distribution of MCNs with different copolymer ratios, the tumor distribution of DiR MCNs with different copolymer ratios was investigated by near-infrared fluorescence (NIRF) imaging. Figure 7A shows the in vivo NIRF images of MCF-7 tumor-bearing mice at different time points after IV injection of DiR MCNs with different copolymer ratios (the loaded DiR concentration in all groups was $10 \mu \mathrm{g} / \mathrm{mL}$ ). After the injection of different MCNs, a strong fluorescence of DiR in the tumor region was observed in all groups (Figure 7B) and achieved the strongest fluorescence intensity at $24 \mathrm{~h}$. Most interestingly while maintaining the same amount of $\mathrm{DiR}$, the highest copolymer ratio group exhibited the highest and longest tumor targeting. A recent study showed that DiR existed in a nonquenched state in the cores of polymeric micelles ${ }^{40}$. Moreover, with the increase of copolymer ratio, it was more difficult for DiR to leak out from MCNs. As a result, the group with the highest copolymer ratio showed the most localization in the tumor region via the EPR effect. Furthermore, consistent with the in vitro cellular uptake assay, the results also demonstrated that, with the increase of copolymer ratio, the clearance rate decreased. After $96 \mathrm{~h}$ of injection, DiR MCNs were still found in the peripheral region of tumor in 3000:1 (MCNs: DiR) group. Increased copolymer ratio created a stronger stealth system, which prevented the tumor cell uptake of DiR and thus slowed down the clearance rate. In another perspective, our results also suggested that the polymer and dye ratio should be optimized when using this delivery system to provide a basis for enhancing surgical guidance via NIR visualization of tumors. Although a higher polymer ratio would slow down the clearance rate, it would also amplify the tumor region at the same time due to their slow internalization and peripheral tumor distribution.

\section{Conclusion}

Understanding the underlying endocytosis mechanism of miktoarm copolymer micelles is critical for the design of non-linear copolymers as effective drug carriers. Despite numerous studies examining the endocytosis and intracellular trafficking of polymeric micelles ${ }^{26,38,41}$, whether the endocytosis mechanism discovered in vitro can be applied in vivo remains unknown. This study is essential considering that, only by confirming the in vitro-in vivo correlation of copolymer nanomicelles in cellular uptake and trafficking could we then validate and apply the mechanism discovered in the cellular level to design new copolymers for drug delivery. Our findings demonstrated 
that the in vitro and in vivo mechanism of cellular uptake and intracellular trafficking of MCNs involved a clear and similar sequence of events: interaction of MCNs with the cell membrane induced hyperbranched block PCL embedding into the cytomembrane, which resulted in the release of loaded hydrophobic fluorescence probes from the cores of MCNs and was followed by time-dependent intracellular clustering within endocytic vesicles. Moreover, the uptake amount and speed of loaded fluorescent probes were correlated with the mass ratios of MCNs in vitro and in vivo. A high mass ratio of MCNs to loaded hydrophobic probes also resulted in slower clearance rate of loaded fluorescent probes in the peripheral region of the tumor. This study not only highlights the need for more thorough studies regarding the in vitro-in vivo transport mechanism correlation of polymeric nanomaterials but also provides a universal method of visualizing this relationship both in vitro and in vivo. Continued research in this topic would help the design of more effective nanomaterials platforms for biomedical applications. The current work initiates the impetus to synthesize more kinds of hyperbranched copolymers to navigate the characteristics of the cell membrane for better cellular targeting and penetrating. 


\section{Figure legend}

Figure 1. (A) Schematic illustration of miktoarm copolymer nanomicelles (MCNs), made of $P_{E G}$ 113- $(h b-P G)_{15}-g-P C L_{22}$. (B) Schematic illustration of the concept of the study. Using the technique of Förster resonance energy transfer (FRET), the cellular uptake and trafficking behavior can be monitored.

Figure 2. Fluorospectra and confocal laser scanning microscopy (CLSM) image of MCNs. (A) Fluorospectra of MCNs loaded with DiO (DiO MCNs), MCNs loaded with Dil (Dil MCNs), MCNs co-loaded with $\mathrm{DiO}$ and Dil (DiO/Dil MCNs), mixtures of MCNs loaded with $\mathrm{DiO}$ and MCNs loaded with Dil (DiO MCNs \& Dil MCNs) and DiO/Dil MCNs decomposed by acetonitrile (Destroyed DiO/Dil MCNs). (B) CLSM image of DiO/Dil MCNs and acetonitrile destroyed DiO/Dil MCNs. Green fluorescence represents the fluorescence of DiO. Red fluorescence represents the fluorescence of Dil. The concentration of DiO was $2 \mu \mathrm{g} / \mathrm{mL}$, the concentration of Dil was $2 \mu \mathrm{g} / \mathrm{mL}$ for all formulations that has $\mathrm{DiO}$ or Dil.

Figure 3. Intracellular trafficking studies of DiO/Dil MCNs and DiO MCNs \& Dil MCNs Mixtures to MCF-7 cells via CLSM. (A) CLSM images of MCF-7 cells incubated with 1.5 $\mathrm{mg} / \mathrm{mL} \mathrm{DiO} / \mathrm{Dil} \mathrm{MCNs}$ (loaded with $0.16 \% \mathrm{DiO}$ and $0.11 \% \mathrm{Dil}$ ). (B) CLSM images of MCF-7 cells incubated with $1.5 \mathrm{mg} / \mathrm{mL}$ DiO MCNs \& Dil MCNs Mixtures (loaded with $0.16 \% \mathrm{DiO}$ and $0.11 \%$ Dil). (Scale bar: $25 \mu \mathrm{m}$ ) (C) FRET efficiencies calculated from CLSM images with FRET SE method $(n=30)$. Green fluorescence represents the fluorescence of DiO. Red fluorescence represents the fluorescence of Dil.

Figure 4. Fluorospectra and in vivo NIR fluorescence imaging of MCNs. (A) Fluorospectra of MCNs loaded with DiD (DiD MCNs), MCNs loaded with DiR (DiR MCNs), MCNs co-loaded with DiD and DiR (DiD/DiR MCNs), mixtures of MCNs loaded with DiD and MCNs loaded with DiR (DiD MCNs \& DiR MCNs Mixtures). (B) In vivo NIR fluorescence imaging of of DiD/DiR MCNs and DiD MCNs \& DiR MCNs Mixtures.

Figure 5. In vivo fate of FRET MCNs on MCF-7 tumor-bearing mice. (A) NIR fluorescence imaging of MCF-7 tumor-bearing mice at 1, 3, 5, 8, 12 and $24 \mathrm{~h}$ after iv injection of DiD/DiR MCNs and DiD MCNs \& DiR MCNs Mixtures. (B) CLSM images of fluorescence detection of $\mathrm{DiO}$ and Dil in tumor cryosections after iv injection of $\mathrm{DiD} / \mathrm{DiR}$ MCNs and DiD MCNs \& DiR MCNs Mixtures.

Figure 6. Cellular uptake of MCNs with different copolymer ratio. (A) Fluorescence intensity of MCF-7 cells analyzed by flow cytometric after treated with DiO/Dil MCNs at different copolymer ratios to probes: 500:1 (Aa), 750:1 (Ab), 1500:1 (Ac), 3000:1(Ad) at 1(A1), 2 (A2), 4h (A3). Green represents the fluorescence of DiO. Red represents the fluorescence of Dil. (B) Uptake speed of DiO (B1) and Dil (B2) at different copolymer ratios to probes: 500:1, 750:1 1500:1. (C) CLSM images of fluorescence detection of $\mathrm{DiO}$ and Dil in MCF-7 cells with DiO/Dil MCNs at different copolymer ratios to probes: $500: 1,750: 1,1500: 1,3000: 1$ at 4 h. (D) Flow cytometry analysis for MCNs loaded with $\mathrm{DiO}(\mathrm{Db})$ and $\mathrm{Dil}(\mathrm{Dc})$ without preincubation (D1) or with preincubation (D2) of blank 
MCNs. The concentration of DiO was $2 \mu \mathrm{g} / \mathrm{mL}$, the concentration of Dil was $2 \mu \mathrm{g} / \mathrm{mL}$ for all formulations that has $\mathrm{DiO}$ or Dil.

Figure 7. In vivo NIR fluorescence imaging of MCF-7 tumor bearing mice at the indicated time point after iv injection of DiR MCNs with different ratio of copolymers. (A) NIR fluorescence imaging of DiR MCNs with different ratio of copolymers. (B) In vivo real-time imaging of MCF-7 tumor bearing mice. The concentration of DiR was 10 $\mu \mathrm{g} / \mathrm{mL}$ for all formulations at a dose of $100 \mu \mathrm{g} / \mathrm{kg}$.

Table S1. Characteristics of $\mathrm{PEG}_{113}-\left(h b-P G_{15}-g-\mathrm{PCL}_{22}\right.$ miktoarm copolymer nanomicelles

Figure S1. Results of the cell viability after being treated with MCNs with different concentration, determined by $(A)$ the SRB assay $(n=5)$ and $(B)$ the LDH assay $(n=3)$.

Figure S2. CLSM image of MCF-7 cells incubated with DiO/Dil MCNs for 4 h. (A) Images obtained to calculate FRET efficiencies by FRET Sensitized Emission (SE) method. (B) Images obtained to calculate FRET efficiencies by FRET Acceptor PhotoBleaching $(A B)$ method. Circled region was photobleaching points. Five points were selected to be bleached accordingly. Green fluorescence represents the fluorescence of DiO. Red fluorescence represents the fluorescence of Dil. (Scale bar: $25 \mu \mathrm{m})$ 


\section{Present address}

c (Z.C.) Department of Pharmaceutical Sciences, College of Pharmacy, University of Kentucky, Lexington, Kentucky 40536, USA

d (X.Z.) Department of Computer Science, College of Engineering, University of Kentucky, Lexington, Kentucky 40506, USA

e (S.H.) Medical College, Henan University of Science \& Technology, Luoyang, Henan 471003, China

$f$ (W.G.) Department of Pharmaceutical Sciences, College of Pharmacy, University of Michigan, Ann Arbor, Michigan 48109, USA

\section{Corresponding author}

*(Q. Z.) Email: zqdodo@bjmu.edu

\section{Author contributions}

The manuscript was written through contributions of all authors. All authors have given approval to the final version of the manuscript.

\section{Notes}

The authors declare no competing financial interest.

\section{Acknowledgment}

This work was supported by the Fundamental Research Funds for the National Natural Science Foundation of China (81821004 and 81690264 to Q. Zhang).

\section{Reference}

1. Kim, B. Y.; Rutka, J. T.; Chan, W. C., Nanomedicine. N Engl J Med 2010, 363 (25), 2434-43.

2. Peer, D.; Karp, J. M.; Hong, S.; Farokhzad, O. C.; Margalit, R.; Langer, R., Nanocarriers as an emerging platform for cancer therapy. Nat Nanotechnol 2007, 2 (12), 751-60.

3. Hubbell, J. A.; Chilkoti, A., Chemistry. Nanomaterials for drug delivery. Science 2012, 337 (6092), $303-5$.

4. Zhang, X.; Cui, Z.; Zhang, X.; He, B.; Wang, X.; Zhang, H.; Zhong, Z.; Zhang, Q., Characteristics, cellular uptake and transepithelial transport of coumarin-6 loaded PEO-(hb-PG)-g-PCL miktoarm copolymer micelles. J. Chin. Pharm. Sci. 2013, 22 (3).

5. $\quad$ Cagel, M.; Tesan, F. C.; Bernabeu, E.; Salgueiro, M. J.; Zubillaga, M. B.; Moretton, M. A.; Chiappetta, D. A., Polymeric mixed micelles as nanomedicines: Achievements and perspectives. European Journal of Pharmaceutics and Biopharmaceutics 2017, 113, 211-228.

6. Kim, K. S.; Park, W.; Hu, J.; Bae, Y. H.; Na, K., A cancer-recognizable MRI contrast agents using pHresponsive polymeric micelle. Biomaterials 2014, 35 (1), 337-343.

7. Hui, L.; G., R. P.; Zheng, C.; Fengmei, P.; Mark, E. B.; Dan, S.; Peixuan, G.; Wei, L., RNA Nanoparticles Derived from Three-Way Junction of Phi29 Motor pRNA Are Resistant to I-125 and Cs-131 Radiation. Nucleic Acid Therapeutics 2015, 25 (4), 188-197.

8. Trubetskoy, V. S.; Torchilin, V. P., Use of polyoxyethylene-lipid conjugates as long-circulating carriers for delivery of therapeutic and diagnostic agents. Advanced drug delivery reviews 1995, 16 (2-3), 311-320.

9. Jin, X.; Sun, P.; Tong, G.; Zhu, X., Star polymer-based unimolecular micelles and their application in bioimaging and diagnosis. Biomaterials 2018, 178, 738-750.

10. Chen, S.; Zhang, X. Z.; Cheng, S. X.; Zhuo, R. X.; Gu, Z. W., Functionalized amphiphilic hyperbranched polymers for targeted drug delivery. Biomacromolecules 2008, 9 (10), 2578-85.

11. Gaballa, H.; Theato, P., Glucose-Responsive Polymeric Micelles via Boronic Acid-Diol Complexation for 
Insulin Delivery at Neutral pH. Biomacromolecules 2019, 20 (2), 871-881.

12. Zhu, Y.; Meng, T.; Tan, Y.; Yang, X.; Liu, Y.; Liu, X.; Yu, F.; Wen, L.; Dai, S.; Yuan, H.; Hu, F., Negative Surface Shielded Polymeric Micelles with Colloidal Stability for Intracellular Endosomal/Lysosomal Escape. Molecular pharmaceutics 2018, 15 (11), 5374-5386.

13. Jain, R. K.; Stylianopoulos, T., Delivering nanomedicine to solid tumors. Nature reviews. Clinical oncology 2010, 7 (11), 653-64.

14. Murakami, M.; Cabral, H.; Matsumoto, Y.; Wu, S.; Kano, M. R.; Yamori, T.; Nishiyama, N.; Kataoka, K., Improving drug potency and efficacy by nanocarrier-mediated subcellular targeting. Science translational medicine 2011, 3 (64), 64ra2.

15. Wang, Y.; Grayson, S. M., Approaches for the preparation of non-linear amphiphilic polymers and their applications to drug delivery. Advanced drug delivery reviews 2012, 64 (9), 852-865.

16. Bates, F. S.; Hillmyer, M. A.; Lodge, T. P.; Bates, C. M.; Delaney, K. T.; Fredrickson, G. H., Multiblock polymers: panacea or Pandora's box? Science 2012, 336 (6080), 434-40.

17. Zhang, X.; Cheng, J.; Wang, Q.; Zhong, Z.; Zhuo, R., Miktoarm Copolymers Bearing One Poly(ethylene glycol) Chain and Several Poly( $\varepsilon$-caprolactone) Chains on a Hyperbranched Polyglycerol Core. Macromolecules 2010,43 (16), 6671-6677.

18. Prencipe, G.; Tabakman, S. M.; Welsher, K.; Liu, Z.; Goodwin, A. P.; Zhang, L.; Henry, J.; Dai, H., PEG branched polymer for functionalization of nanomaterials with ultralong blood circulation. J Am Chem Soc 2009, 131 (13), 4783-7.

19. Matsumura, Y., Preclinical and clinical studies of NK012, an SN-38-incorporating polymeric micelles, which is designed based on EPR effect. Adv Drug Deliv Rev 2011, 63 (3), 184-92.

20. Matsumura, Y.; Kataoka, K., Preclinical and clinical studies of anticancer agent-incorporating polymer micelles. Cancer Sci 2009, 100 (4), 572-9.

21. Yokoyama, M., Polymeric micelles as a new drug carrier system and their required considerations for clinical trials. Expert Opin Drug Deliv 2010, 7 (2), 145-58.

22. Deng, C.; Jiang, Y.; Cheng, R.; Meng, F.; Zhong, Z., Biodegradable polymeric micelles for targeted and controlled anticancer drug delivery: Promises, progress and prospects. Nano Today 2012, 7 (5), $467-480$.

23. Zhao, S.; Dai, W.; He, B.; Wang, J.; He, Z.; Zhang, X.; Zhang, Q., Monitoring the transport of polymeric micelles across MDCK cell monolayer and exploring related mechanisms. Journal of controlled release 2012, 158 (3), 413-423.

24. Deng, F.; Yu, C.; Zhang, H.; Dai, W.; He, B.; Zheng, Y.; Wang, X.; Zhang, Q., The effect of hydrophilic and hydrophobic structure of amphiphilic polymeric micelles on their transportation in rats. Current drug delivery 2016, 13 (1), 105-110.

25. Vercauteren, D.; Vandenbroucke, R. E.; Jones, A. T.; Rejman, J.; Demeester, J.; De Smedt, S. C.; Sanders, N. N.; Braeckmans, K., The use of inhibitors to study endocytic pathways of gene carriers: optimization and pitfalls. Molecular therapy : the journal of the American Society of Gene Therapy 2010, 18 (3), 561-9.

26. Moghimi, S. M.; Hunter, A. C.; Murray, J. C.; Szewczyk, A., Cellular distribution of nonionic micelles. Science 2004, 303 (5658), 626-8; author reply 626-8.

27. Wang, X.; Wang, Y.; Chen, X.; Wang, J.; Zhang, X.; Zhang, Q., NGR-modified micelles enhance their interaction with CD13-overexpressing tumor and endothelial cells. Journal of controlled release : official journal of the Controlled Release Society 2009, 139 (1), 56-62.

28. Zhang, J.-Y.; He, B.; Qu, W.; Cui, Z.; Wang, Y.-b.; Zhang, H.; Wang, J.-C.; Zhang, Q., Preparation of the albumin nanoparticle system loaded with both paclitaxel and sorafenib and its evaluation in vitro and in vivo. Journal of Microencapsulation 2011, 28 (6), 528-536.

29. Zhuang, J.; Ping, Q.; Song, Y.; Qi, J.; Cui, Z., Effects of chitosan coating on physical properties and pharmacokinetic behavior of mitoxantrone liposomes. International journal of nanomedicine 2010, 5, 407-16.

30. Gao, W.; Lin, Z.; Chen, M.; Yang, X.; Cui, Z.; Zhang, X.; Yuan, L.; Zhang, Q., The co-delivery of a low-dose Pglycoprotein inhibitor with doxorubicin sterically stabilized liposomes against breast cancer with low P-glycoprotein expression. International journal of nanomedicine 2014, 9, 3425-37.

31. He, S.; Cui, Z.; Mei, D.; Zhang, H.; Wang, X.; Dai, W.; Zhang, Q., A cremophor-free self-microemulsified delivery system for intravenous injection of teniposide: evaluation in vitro and in vivo. AAPS PharmSciTech 2012,13 (3), 846-52.

32. He, S.; Cui, Z.; Wang, X.; Zhang, H.; Dai, W.; Zhang, Q., Cremophor-free intravenous self-microemulsions for 
teniposide: Safety, antitumor activity in vitro and in vivo. International journal of pharmaceutics 2015, 495 (1), 144153.

33. Liu, P.; Ren, J.; Xiong, Y.; Yang, Z.; Zhu, W.; He, Q.; Xu, Z.; He, W.; Wang, J., Enhancing magnetic resonance/photoluminescence imaging-guided photodynamic therapy by multiple pathways. Biomaterials 2019, 199, 52-62.

34. Vichai, V.; Kirtikara, K., Sulforhodamine B colorimetric assay for cytotoxicity screening. Nature protocols 2006, 1 (3), 1112-6.

35. Malvindi, M. A.; Brunetti, V.; Vecchio, G.; Galeone, A.; Cingolani, R.; Pompa, P. P., SiO 2 nanoparticles biocompatibility and their potential for gene delivery and silencing. Nanoscale 2012, 4 (2), 486-495.

36. Ai, H. W.; Hazelwood, K. L.; Davidson, M. W.; Campbell, R. E., Fluorescent protein FRET pairs for ratiometric imaging of dual biosensors. Nature methods 2008, 5 (5), 401-3.

37. Merzlyak, E. M.; Goedhart, J.; Shcherbo, D.; Bulina, M. E.; Shcheglov, A. S.; Fradkov, A. F.; Gaintzeva, A.; Lukyanov, K. A.; Lukyanov, S.; Gadella, T. W.; Chudakov, D. M., Bright monomeric red fluorescent protein with an extended fluorescence lifetime. Nature methods 2007, 4 (7), 555-7.

38. Chen, H.; Kim, S.; Li, L.; Wang, S.; Park, K.; Cheng, J. X., Release of hydrophobic molecules from polymer micelles into cell membranes revealed by Forster resonance energy transfer imaging. Proceedings of the National Academy of Sciences of the United States of America 2008, 105 (18), 6596-601.

39. Xiao, K.; Luo, J.; Fowler, W. L.; Li, Y.; Lee, J. S.; Xing, L.; Cheng, R. H.; Wang, L.; Lam, K. S., A self-assembling nanoparticle for paclitaxel delivery in ovarian cancer. Biomaterials 2009, 30 (30), 6006-16.

40. Cho, H.; Indig, G. L.; Weichert, J.; Shin, H. C.; Kwon, G. S., In vivo cancer imaging by poly(ethylene glycol)-bpoly(varepsilon-caprolactone) micelles containing a near-infrared probe. Nanomedicine 2012, 8 (2), $228-36$.

41. Savic, R.; Luo, L.; Eisenberg, A.; Maysinger, D., Micellar nanocontainers distribute to defined cytoplasmic organelles. Science 2003, 300 (5619), 615-8. 
Figure 1
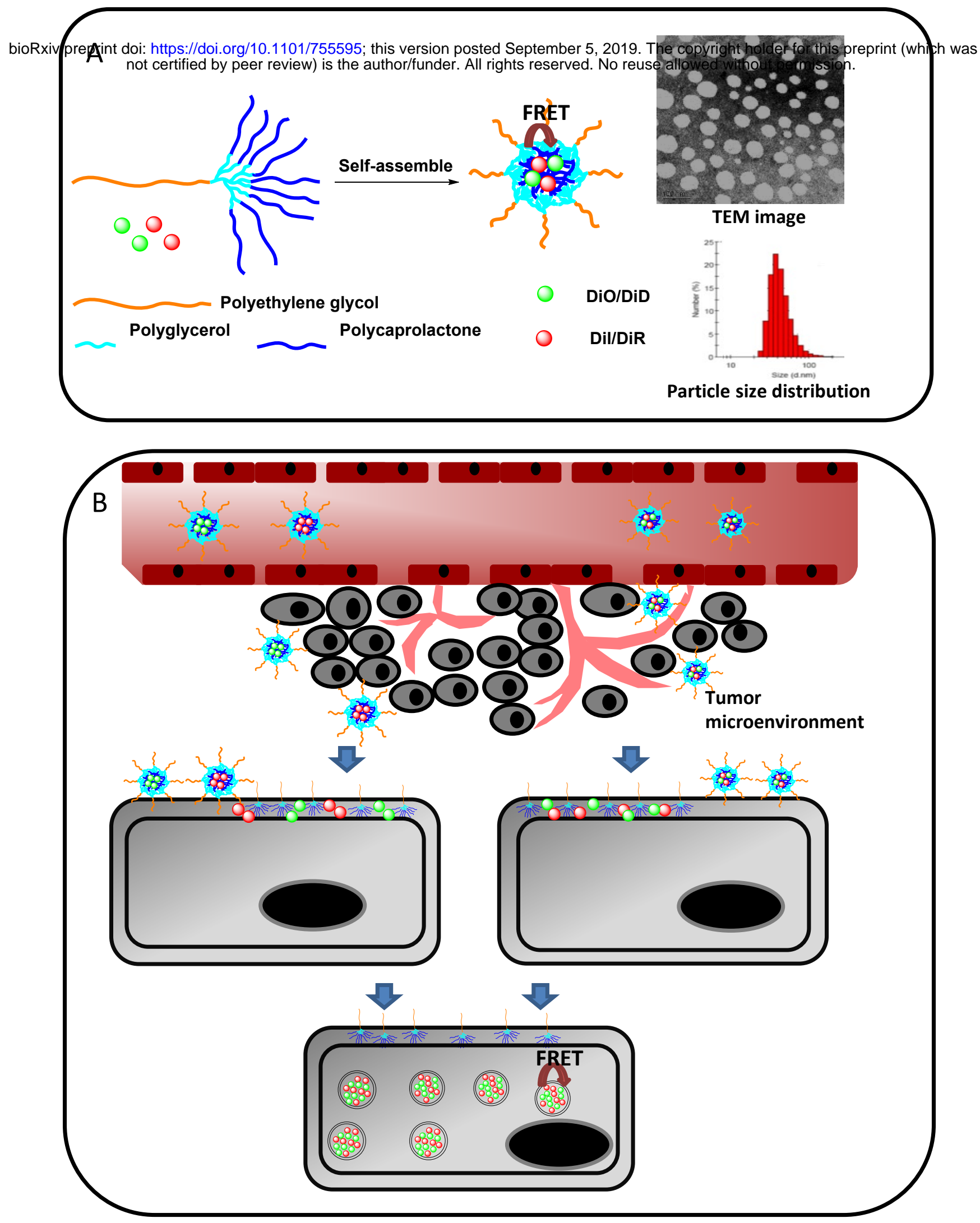
Figure 2

bioRy/ preprint doi: https://doi.org/10.1101/755595; this version posted September 5, 2019. The copyright hyder for this preprint (which was A not certified by peer review) is the author/funder. All rights reserved. No reuse allowed withou permission.

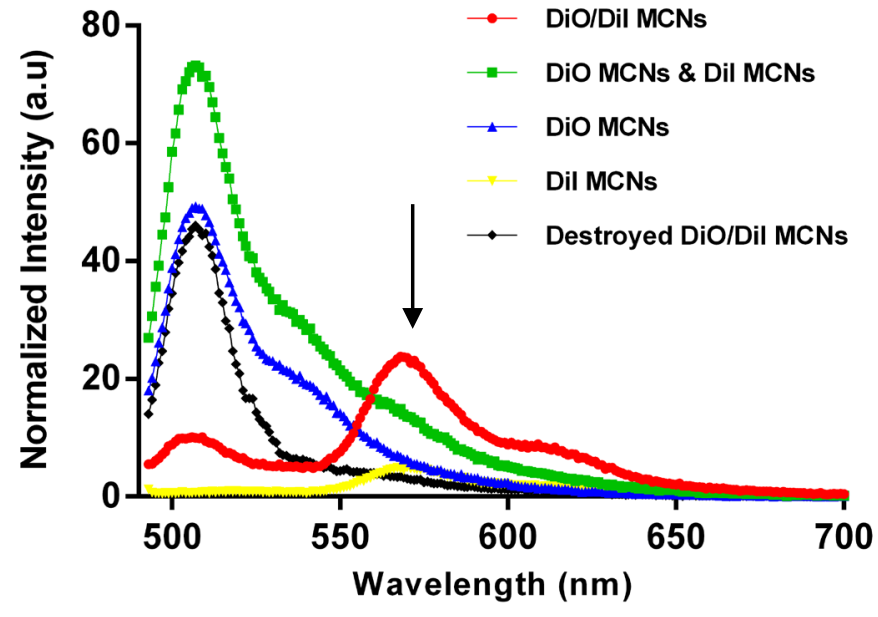

B

DiO

Dil

(Em 501nm)

DiO/Dil MCNs

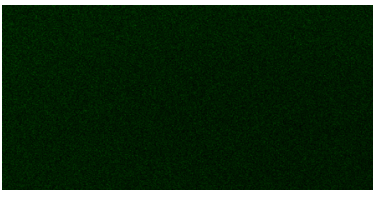

(Em 565nm)

Destroyed

DiO/Dil MCNs
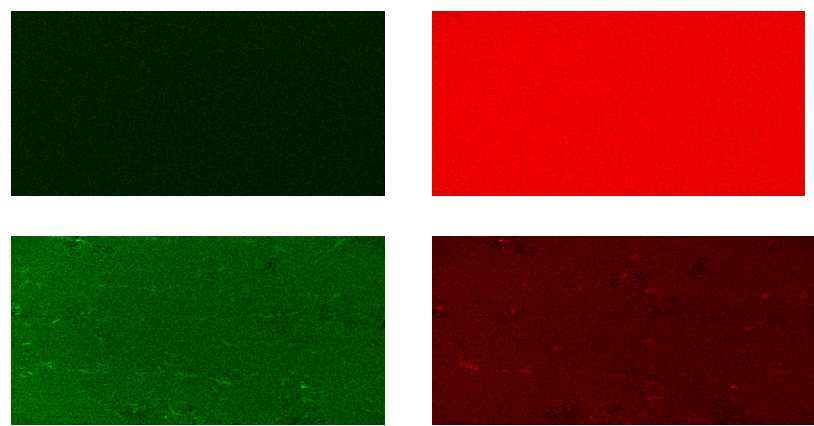
Figure 3

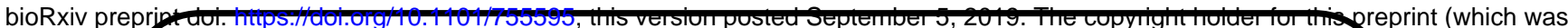
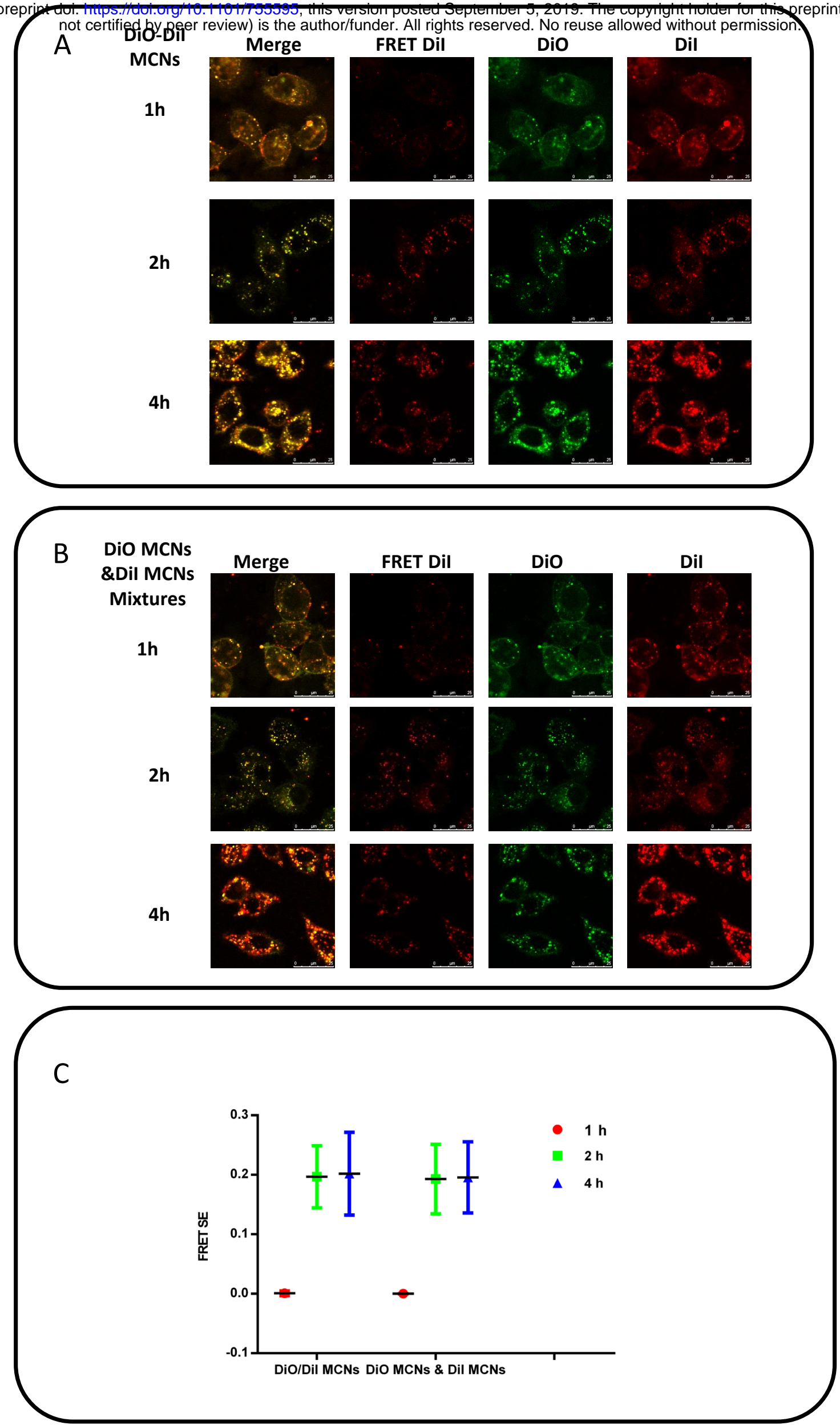
Figure 4

bioRxiv preprint doi: https://doi.org/10.1101/755595; this version posted September 5, 2019. The copyright holder for this preprint (which was not certified by peer review) is the author/funder. All rights reserved. No reuse allowed without permission.
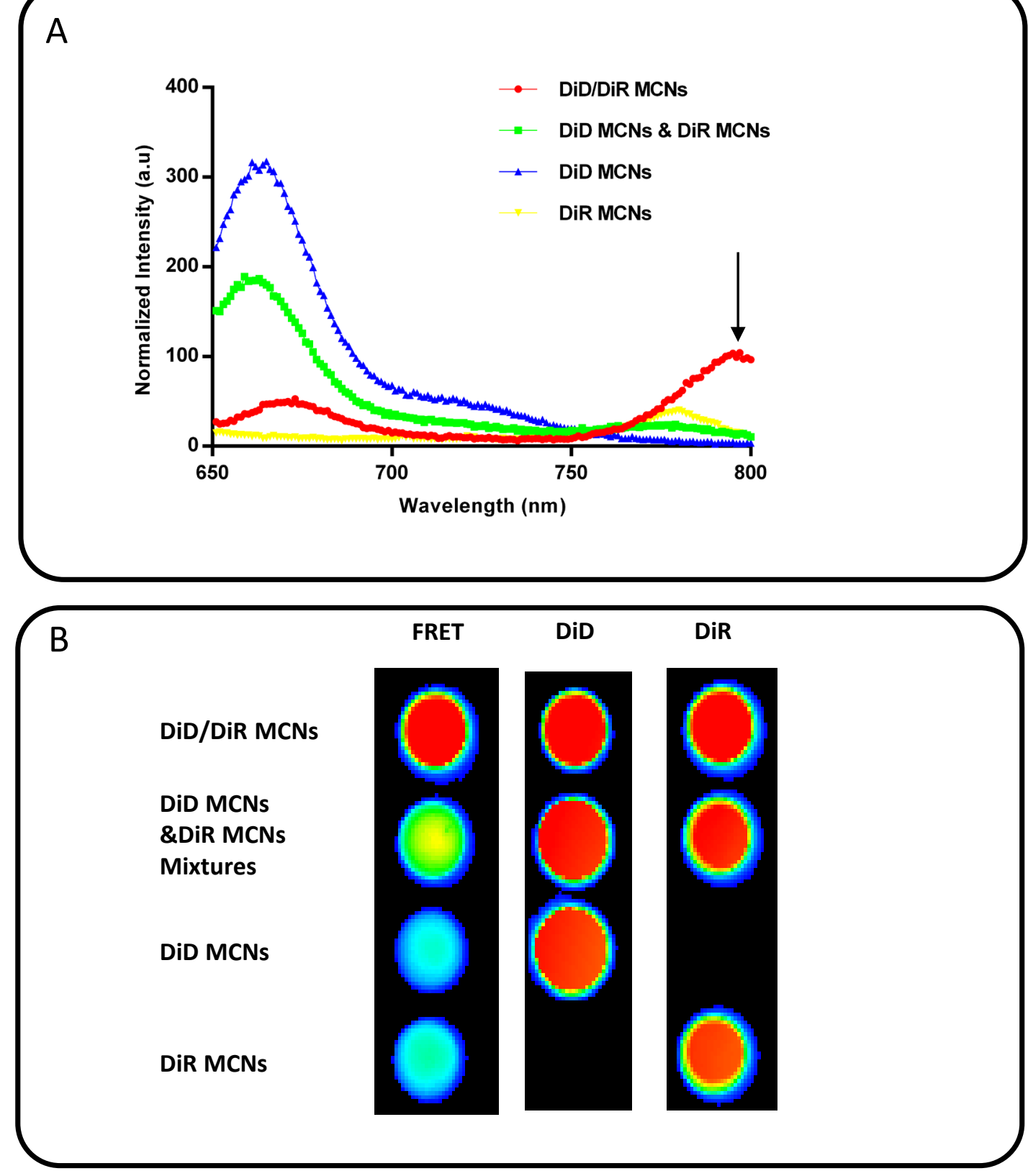


\section{Figure 5}

A

bic Rxiv preprint doi: https://doFRETl Châhing595; this ver\$ion posted September 5, 2019. The copyright holder for this preprint which was not certified by peer review) is the author/funder. All rights reserved. No reuselallowed without permission.

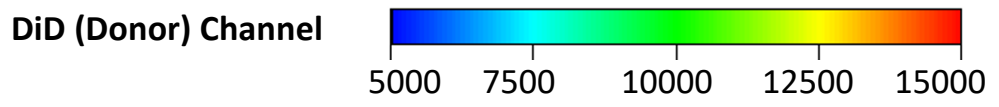

DiR

Channel

(Acceptor)
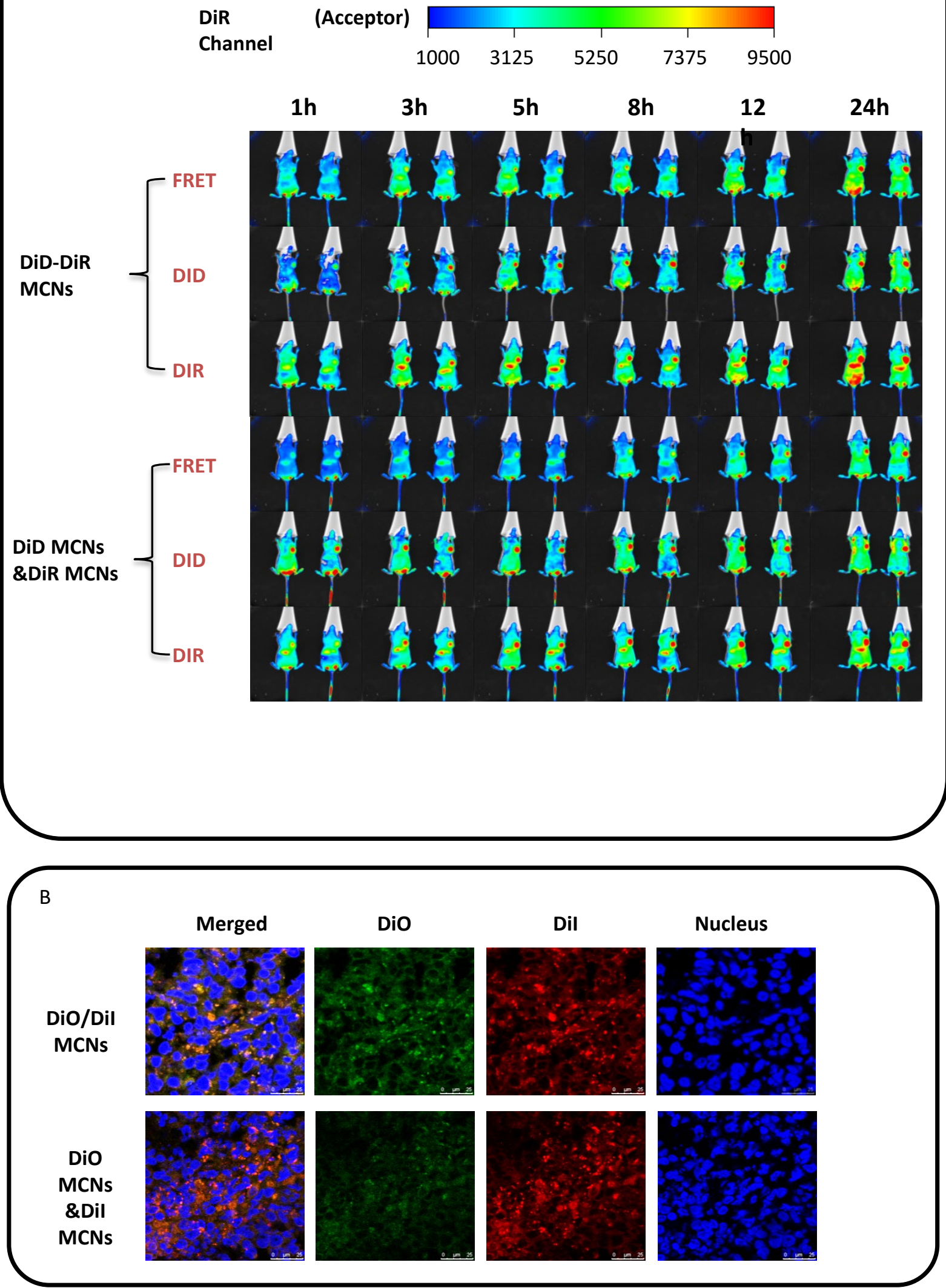
Figure 6

bioR tiv preprint doi: https.

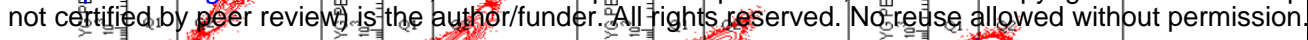

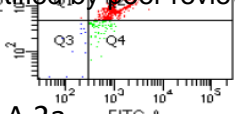

A $2 a^{10^{2}} \stackrel{10^{3}}{ }$ FITC-A

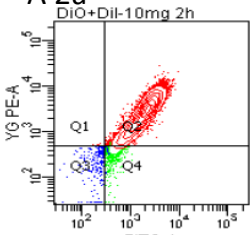

A $3 a$ FITC-A

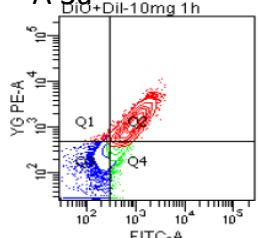

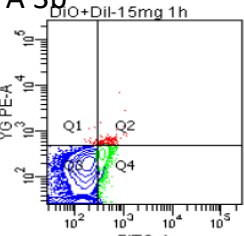

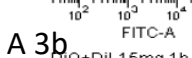

A $2 b^{10^{2}} \stackrel{10^{3}}{F^{2}}{ }^{10^{4}}$

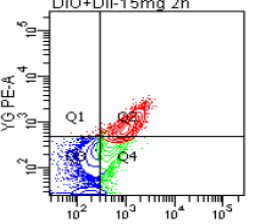

A $2 \mathrm{c}^{10^{2}} \quad 0^{0^{3}} \quad 0^{4}$

A 2c $\mathrm{C}^{10^{2}}{ }^{10} \mathrm{FITC}^{10^{4}}$

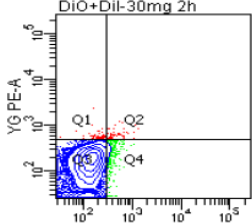

A 3C FITC-A
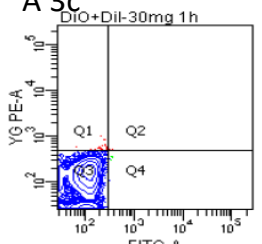

A $2 C^{10^{2}} \stackrel{10^{9}}{\text { FITC }^{10^{4}}}$

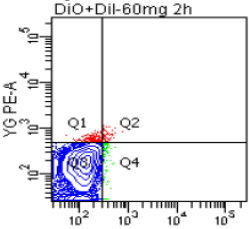

A $3 d^{10^{2}} \stackrel{10}{10} d^{10}-A$

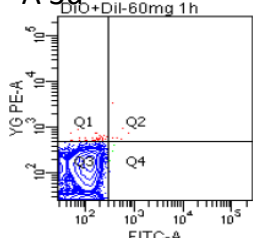

B B1

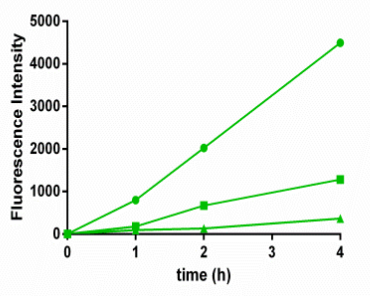

B2

MCNs: DiO=500:1 (w/w) MCNS: DiO=750:1 (w/W) \# MCNs: DiO=1500:1(w/w)

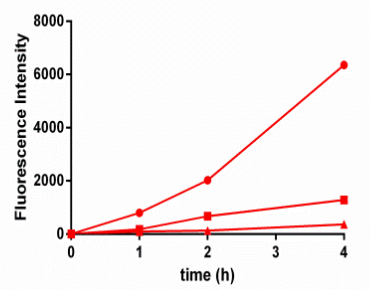

- MCNs: DiO $=500: 1(\mathrm{w} / \mathrm{w}$

- MCNs: DiO $=750: 1(\mathrm{w} / \mathrm{w})$
- MCNs: $\mathrm{DiO}=1500: 1(\mathrm{w} / \mathrm{w})$
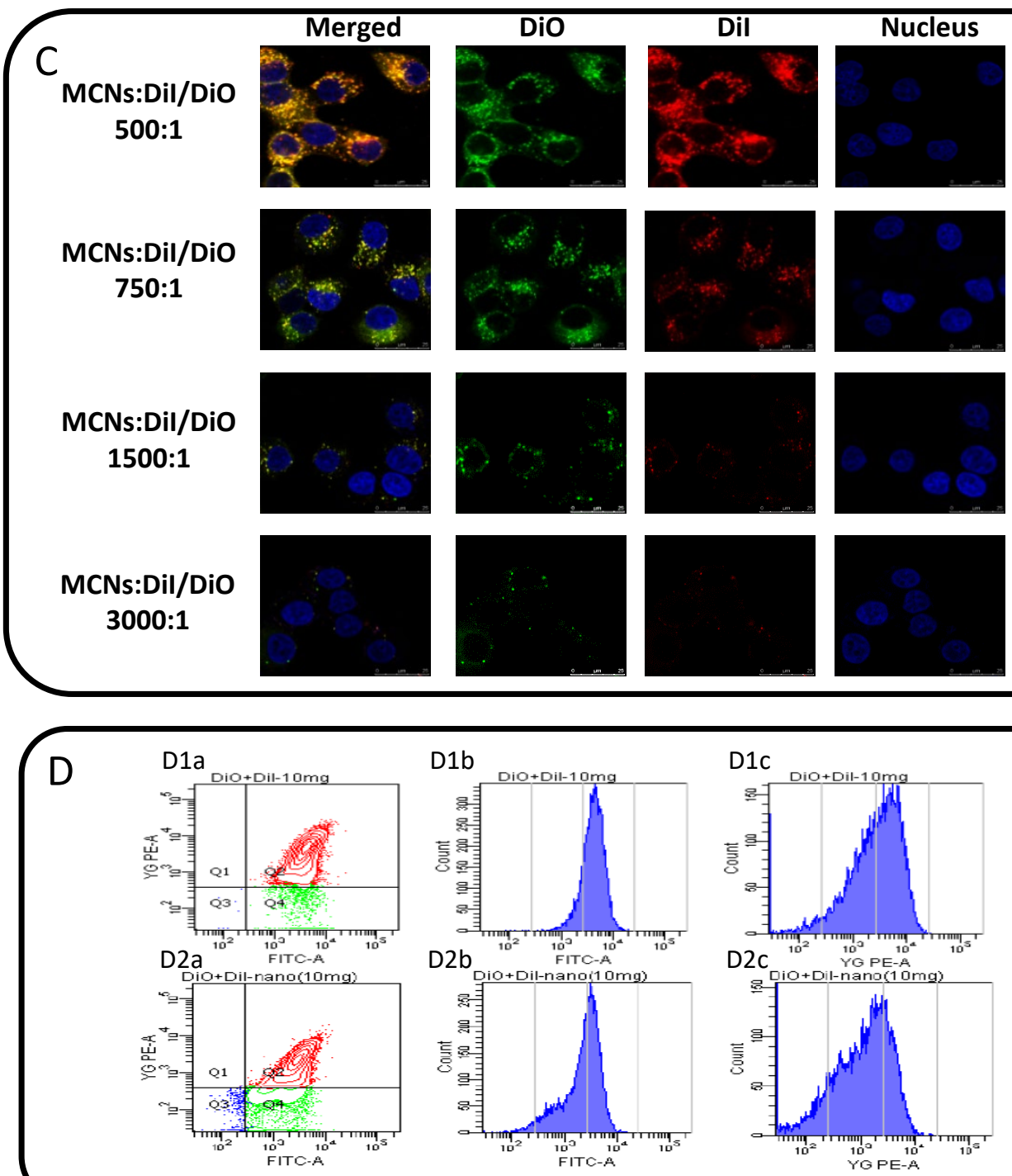
Figure 7

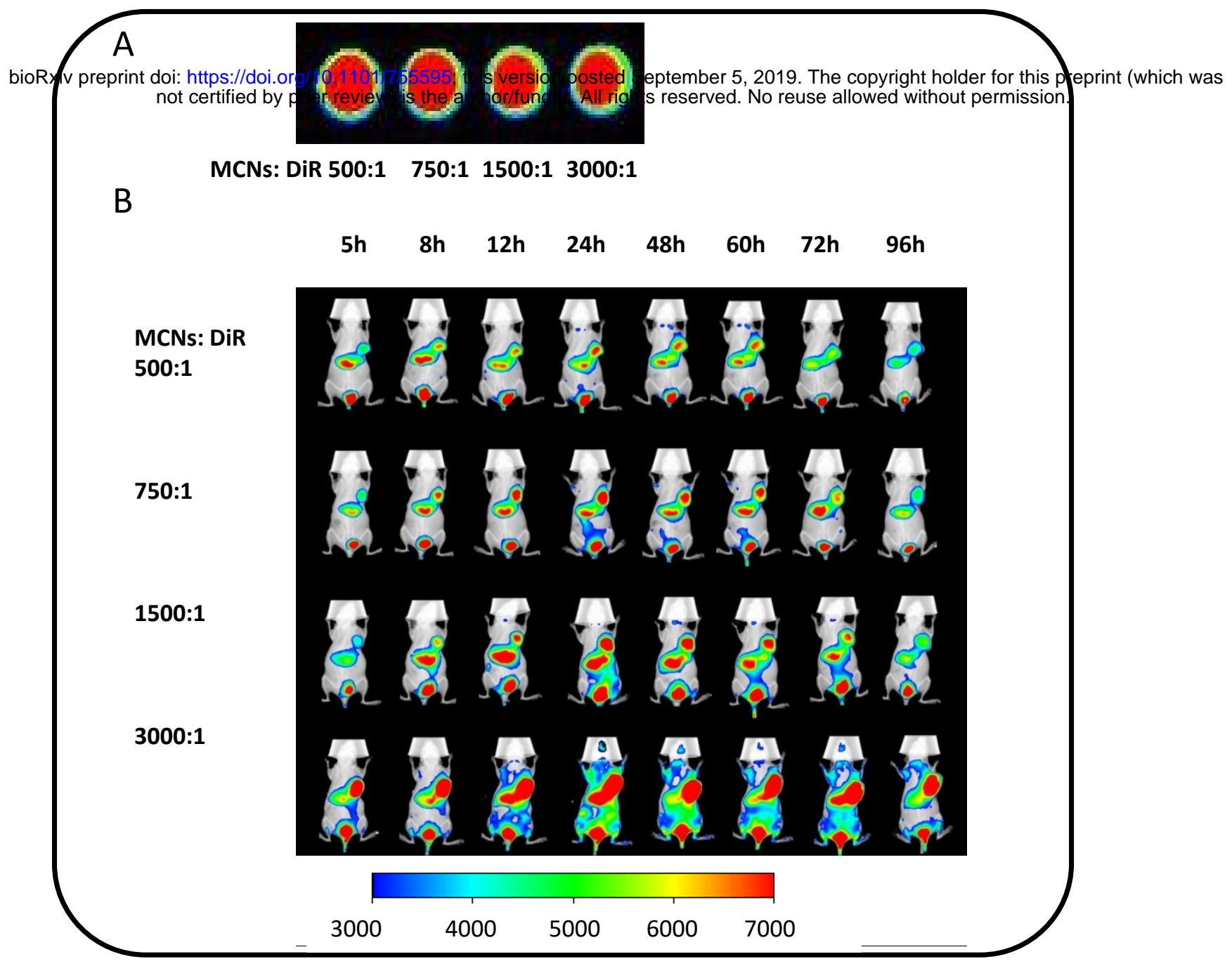




\section{Table S1}

\begin{tabular}{|c|c|c|c|c|}
\hline Preparations & Particle Size (nm) & Polydispersity & Zeta potential(mV) & $\begin{array}{c}\text { Encapsulation } \\
\text { Efficiency(\%) }\end{array}$ \\
\hline MCNs & $45.17 \pm 2.3$ & $0.228 \pm 0.01$ & $-0.013 \pm 0.02$ & - \\
\hline DiO MCNs & $51.41 \pm 4.1$ & $0.16 \pm 0.03$ & $-0.787 \pm 0.11$ & $90.6 \% \pm 0.5$ \\
\hline Dil MCNs & $52.46 \pm 1.2$ & $0.152 \pm 0.02$ & $-1.78 \pm 0.25$ & $92.1 \% \pm 1.2$ \\
\hline DiD MCNs & $55.20 \pm 3.5$ & $0.193 \pm 0.02$ & $-0.925 \pm 0.11$ & $93.4 \% \pm 1.0$ \\
\hline DiR MCNs & $53.31 \pm 3.1$ & $0.219 \pm 0.03$ & $-1.347 \pm 0.21$ & $92.4 \% \pm 0.9$ \\
\hline
\end{tabular}


Figure S1

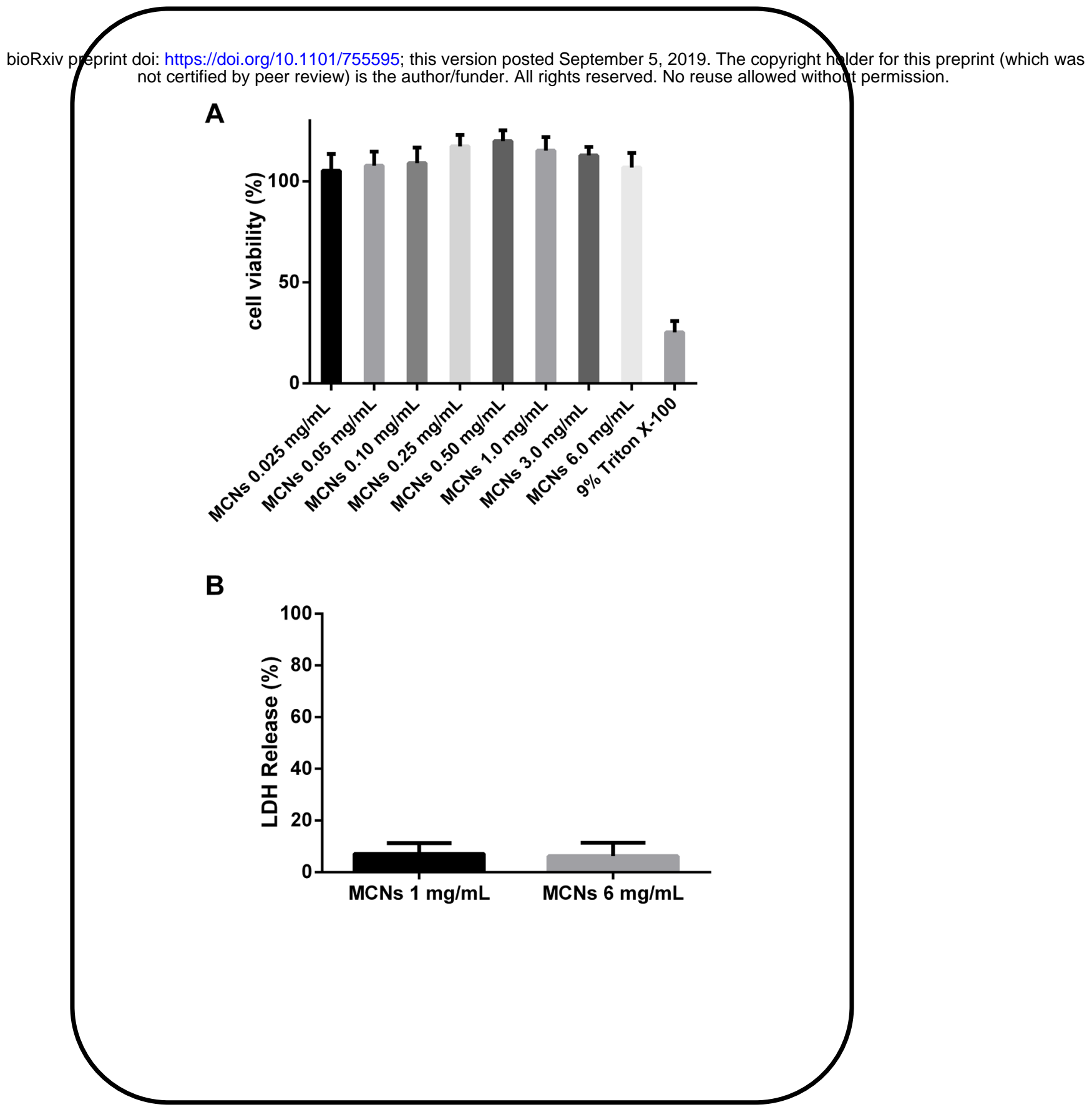


Figure S2

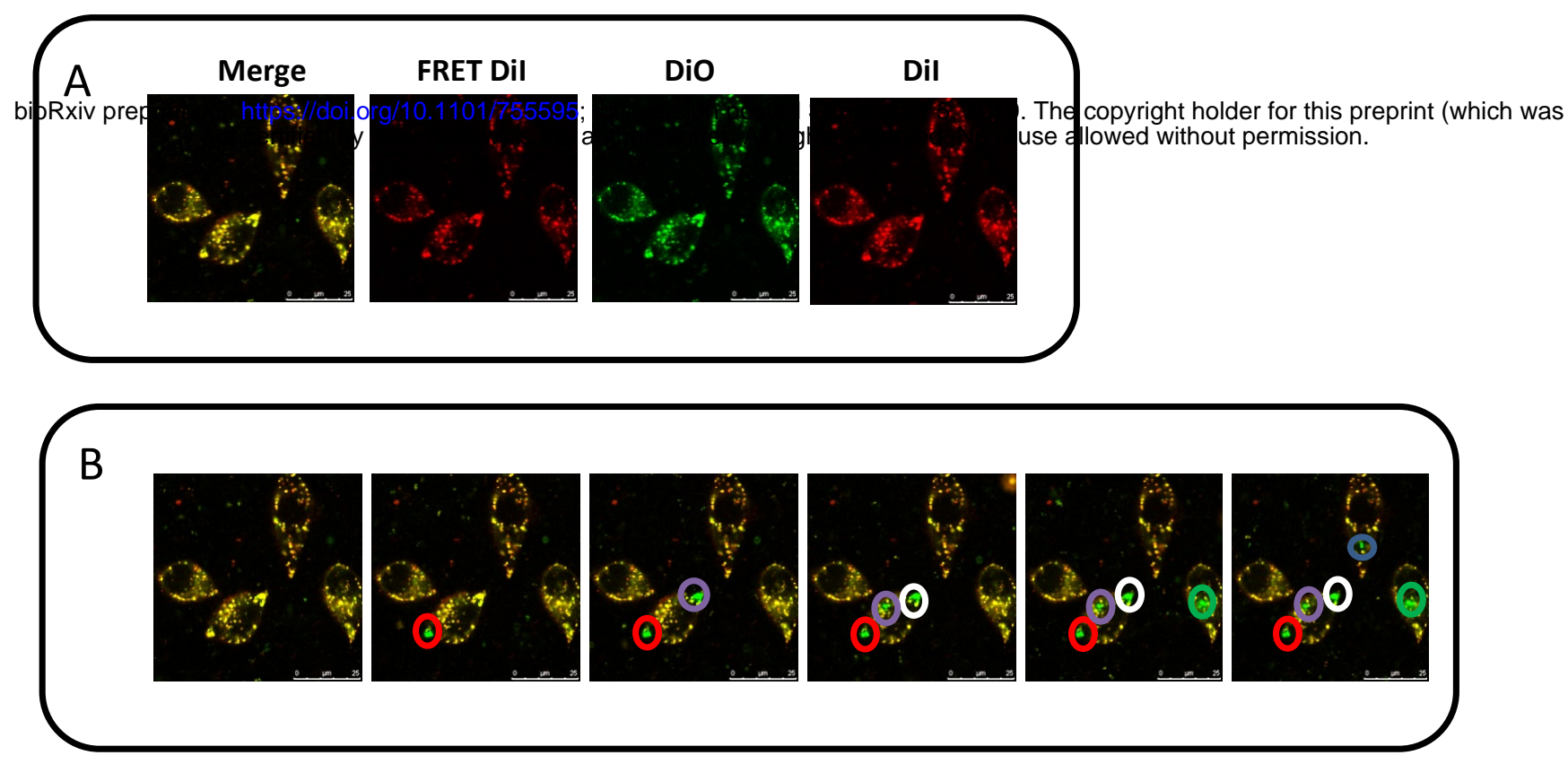

